University of Warwick institutional repository: http://go.warwick.ac.uk/wrap This paper is made available online in accordance with publisher policies. Please scroll down to view the document itself. Please refer to the repository record for this item and our policy information available from the repository home page for further information.

To see the final version of this paper please visit the publisher's website. Access to the published version may require a subscription.

Author(s): P. D. C. King, T. D. Veal, A. Adikimenakis, Hai Lu, L. R. Bailey, E. Iliopoulos, A. Georgakilas, W. J. Schaff, and C. F. McConville Article Title: Surface electronic properties of undoped InAIN alloys Year of publication: 2008 Link to published version: http://dx.doi.org/10.1063/1.2913765

Publisher statement: None 


\title{
Surface electronic properties of undoped InAIN alloys
}

\author{
P. D. C. King, ${ }^{1}$ T. D. Veal, ${ }^{1}$ A. Adikimenakis, ${ }^{2}$ Hai Lu, ${ }^{3, b)}$ L. R. Bailey, ${ }^{1}$ E. Iliopoulos, ${ }^{2}$ \\ A. Georgakilas, ${ }^{2}$ W. J. Schaff, ${ }^{3}$ and C. F. McConville, ${ }^{1, a)}$ \\ ${ }_{1}^{1}$ Department of Physics, University of Warwick, Coventry, CV4 7AL, United Kingdom \\ ${ }^{2}$ Microelectronics Research Group, Department of Physics, University of Crete, P.O. Box 2208, \\ 71003 Heraklion-Crete, Greece \\ ${ }^{3}$ Department of Electrical and Computer Engineering, Cornell University, Ithaca, New York 14853, USA
}

(Received 21 February 2008; accepted 4 April 2008; published online 1 May 2008)

\begin{abstract}
The variation in surface electronic properties of undoped $c$-plane $\operatorname{In}_{x} \mathrm{Al}_{1-x} \mathrm{~N}$ alloys has been investigated across the composition range using a combination of high-resolution $\mathrm{x}$-ray photoemission spectroscopy and single-field Hall effect measurements. For the In-rich alloys, electron accumulation layers, accompanied by a downward band bending, are present at the surface, with a decrease to approximately flatband conditions with increasing $\mathrm{Al}$ composition. However, for the Al-rich alloys, the undoped samples were found to be insulating with approximate midgap pinning of the surface Fermi level observed. () 2008 American Institute of Physics.
\end{abstract} [DOI: 10.1063/1.2913765]

The majority of $n$-type III-V semiconductors investigated to date exhibit a depletion of electrons in the nearsurface region due to the presence of occupied acceptor surface states causing an upward bending of the conduction and valence bands at the surface. In contrast, however, electron accumulation layers occur at InN (Refs. 1 and 2) and InAs (Refs. 3 and 4) surfaces, whereby the Fermi level at the surface is pinned above that of the bulk, resulting in an increase in the near-surface electron concentration compared to the bulk value. The overriding mechanism driving this accumulation of electrons is the particularly low energetic position of the conduction band minimum (CBM), well below the charge neutrality level (CNL) in both materials, resulting in unoccupied, positively charged donor surface states which induce a downward bending of the bands and electron accumulation at the surface. ${ }^{4,5}$ The electron accumulation is still present at the surface of $p$-type material, causing an inversion layer to form where an $n$-type surface region is separated from the $p$-type bulk by a depletion layer. ${ }^{6,7}$

As electron accumulation is uncommon among III-V semiconductors, when $\mathrm{InN}$ is alloyed with other materials, a transition from electron accumulation (inversion) to electron (hole) depletion for $n$-type ( $p$-type) material would be expected. This has previously been investigated for $n$ - and $p$-InGaN via $\mathrm{x}$-ray photoemission spectroscopy (XPS), ${ }^{8-10}$ where such a transition was observed. This was explained by the CNL lying significantly above the CBM for In-rich alloys, but below the CBM for Ga-rich alloys. ${ }^{8-10}$ The band lineup relative to the CNL of the III-N semiconductors is shown in Fig. 1(a). The CNL lies close to the middle of the direct band gap in AlN, similar to the situation in GaN, although much further below the CBM. A similar transition from electron accumulation to depletion may therefore be expected on moving from In-rich to Al-rich $n$-InAlN alloys, motivating the current study. In this letter, high-resolution XPS is utilized to determine the variation of the surface Fermi level pinning position for undoped InAlN alloys

\footnotetext{
${ }^{\text {a) }}$ Electronic mail: c.f.mcconville@warwick.ac.uk.

${ }^{b)}$ Present address: Department of Physics, Nanjing University, Nanjing, 210093, China
}

across the composition range, and the surface electronic properties of these alloys are discussed.

Undoped InAlN alloys were grown by plasma-assisted molecular beam epitaxy on $c$-plane sapphire substrates incorporating AlN buffer layers. The InAlN layer thicknesses ranged from 190 to $4600 \mathrm{~nm}$. The AlN sample was grown to a thickness of $1000 \mathrm{~nm}$ on a $c$-plane sapphire substrate by metal-organic chemical-vapor deposition. Details of the growth methodologies are reported elsewhere. ${ }^{15-17}$ The alloy compositions were determined by x-ray diffraction (XRD) using the AlN (0002) peak as a reference peak. Additionally, Rutherford backscattering (RBS) measurements were performed on the thinnest sample $(190 \mathrm{~nm})$. The composition of this sample was determined to be $\operatorname{In}_{0.60} \mathrm{Al}_{0.40} \mathrm{~N}$ from RBS measurements, in very good agreement with the composition of $\mathrm{In}_{0.59} \mathrm{Al}_{0.41} \mathrm{~N}$ determined from XRD measurements. Highresolution XPS measurements were performed using a Scienta ESCA300 spectrometer at the National Centre for Electron Spectroscopy and Surface Analysis, Daresbury Laboratory, UK. Details of the spectrometer and its arrangement are reported elsewhere. ${ }^{8}$ The binding energy scale is
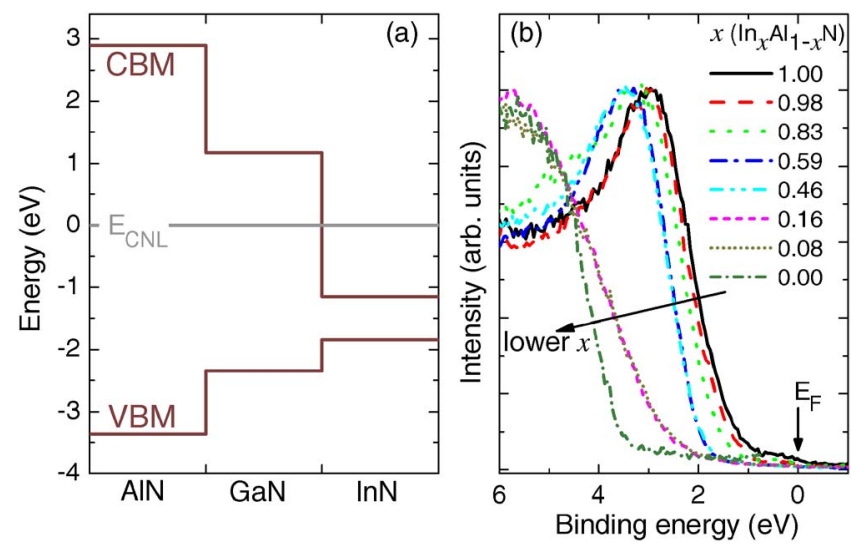

FIG. 1. (Color online) (a) Band lineup relative to the CNL of the III-N semiconductors, determined from measured valence band offsets (Refs. 11 and 12) band gaps (Refs. 13 and 14) and the measured VBM to CNL separation for InN (Ref. 5). (b) Valence band photoemission spectra with respect to the Fermi level $E_{F}$ of undoped $\operatorname{In}_{x} \mathrm{Al}_{1-x} \mathrm{~N}$ alloys across the composition range. 


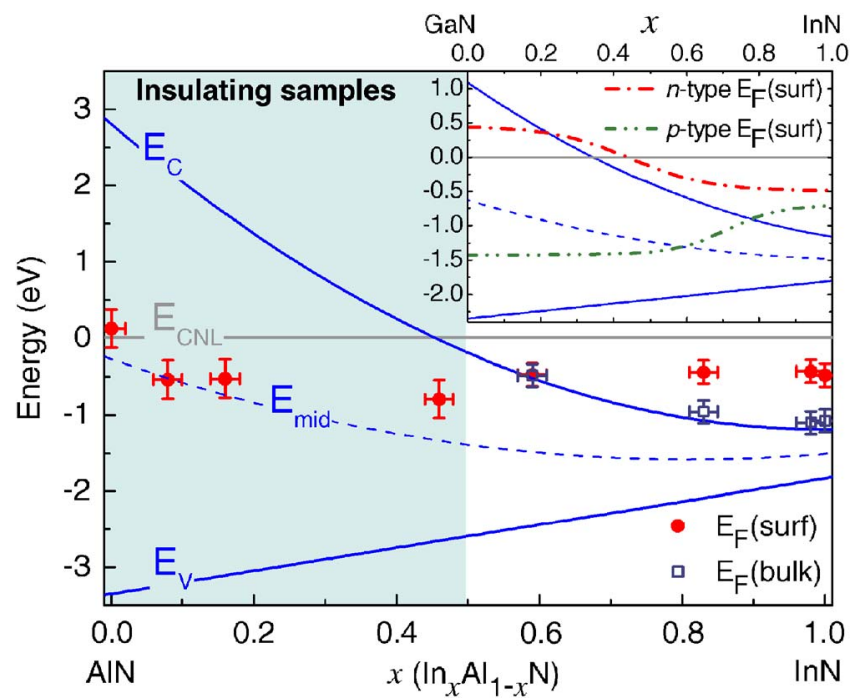

FIG. 2. (Color online) Surface (filled circles) and bulk (open squares) Fermi level $\left(E_{F}\right)$ for undoped $\operatorname{In}_{x} \mathrm{Al}_{1-x} \mathrm{~N}$ alloys relative to the CNL $\left(E_{\mathrm{CNL}}\right)$, and InAlN band edges $\left(E_{C}, E_{V}\right)$ and midgap position $\left(E_{\text {mid }}\right)$. The CNL is located $1.83 \mathrm{eV}$ above the VBM in InN from Ref. 5. The variation in valence band edge is assumed to be linear after Mönch (Ref. 20), with the measured valence band offset of $1.52 \mathrm{eV}$ (Ref. 12) and a band gap bowing parameter of $4 \mathrm{eV}$ (Ref. 21), entirely in the conduction band due to the linear variation of the valence band edge, used to define the band edges relative to the CNL. The equivalent plot for $\operatorname{In}_{x} \mathrm{Ga}_{1-x} \mathrm{~N}$ alloys, adapted from Ref. 10, is shown inset, indicating the general trend in surface Fermi level pinning position for $n$-type (dot-dash line) and $p$-type (dot-dot-dash line) alloys.

given with respect to the Fermi level. For the In-rich samples, this was calibrated using the Fermi edge of an ionbombarded silver reference sample. However, the Al-rich samples were insulating and so required charge compensation via a low-energy electron flood gun. For these alloys, the calibration of the Fermi level was achieved via a referencing of the binding energy of the C $1 s$ core-level peak (from physisorbed carbon) to the average $\mathrm{C} 1 s$ binding energy for the In-rich samples, where no flood gun had been used. The position of the valence band maximum (VBM) at the surface is determined by extrapolating a linear fit of the leading edge of the valence band photoemission to the background level to account for the broadening of the photoemission spectra. ${ }^{18}$

The valence band photoemission is shown in Fig. 1(b). The leading edge of this valence band photoemission shifts to higher binding energies with increasing $\mathrm{Al}$ content, as was previously observed for increasing Ga content in $n$-InGaN alloys, ${ }^{8,10}$ indicating an increase in the VBM to surface Fermi level separation with increasing Al content. The surface Fermi level positions, determined from the XPS measurements, relative to the semiconductor band edges and the $\mathrm{CNL}$ are shown in Fig. 2. Additionally, the carrier density was measured by the single-field Hall effect, and these values were used to calculate (via carrier statistics calculations incorporating a Kane ${ }^{19}$ nonparabolic conduction band) an upper limit of the bulk Fermi level. This is shown in Fig. 2 for the In-rich samples and is an upper limit of the bulk Fermi level as the electron accumulation present for In-rich samples leads to a slight overestimation of the bulk carrier density by single-field Hall effect measurements. The Al-rich samples were insulating by single-field Hall effect measurements, and so, although a precise value of the bulk Fermi level could not be determined, it is expected to lie significantly below the CBM, approaching the midgap position. The insulating nature of the Al-rich samples was confirmed Downloaded 30 Jun 2009 to 137.205.202.8. Redistribution subject

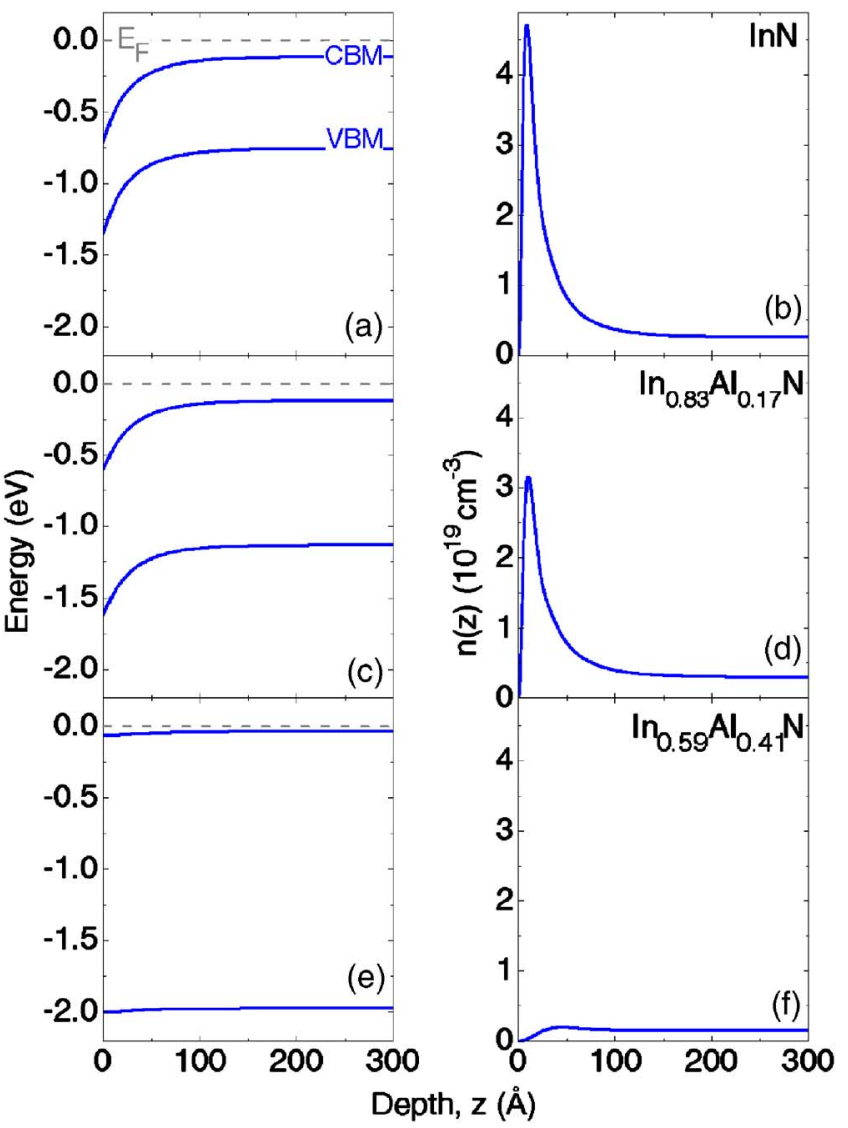

FIG. 3. (Color online) Band bending and carrier concentration profiles as a function of depth from Poisson-MTFA calculations for [(a) and (b)] InN, [(c) and (d)] $\operatorname{In}_{0.83} \mathrm{Al}_{0.17} \mathrm{~N}$, and [(e) and (f) $] \operatorname{In}_{0.59} \mathrm{Al}_{0.41} \mathrm{~N}$.

by the necessity of using a low-energy electron flood gun to achieve charge compensation in the XPS measurements.

For InN, the surface Fermi level lies significantly above the bulk Fermi level, indicating that electron accumulation is present at the surface. This can be understood within the concept of virtual gap states (ViGS): ${ }^{22}$ As the bulk Fermi level lies below the CNL, the surface Fermi level also pins slightly below the CNL, causing a number of donor ViGS to be unoccupied, donating their electrons into the accumulation layer and causing a large downward band bending, as has previously been discussed in detail for $\mathrm{InN}$ accumulation layers. ${ }^{2,5}$ The band bending and carrier concentration profiles in the near-surface region can be calculated from the surface and bulk Fermi levels via a solution of Poisson's equation within a modified Thomas-Fermi approximation $^{23}$ (MTFA) as described elsewhere. ${ }^{24}$ The results of such a calculation are shown in Figs. 3(a) and 3(b) for InN revealing the large downward band bending and extreme electron accumulation present for this sample.

Upon moving to slightly more Al-rich compositions, the surface Fermi level still remains pinned above the bulk Fermi level, indicating electron accumulation is still present at these surfaces. This is consistent with the surface Fermi level lying below the CNL and hence causing some donor ViGS to be unoccupied as for InN. However, the surface and bulk Fermi levels move closer together with increasing $\mathrm{Al}$ content, indicating a reduction in the amount of band bending and hence, a reduction in the degree of electron accumulation at the surface. This is clearly evident from the PoissonMTFA calculations for the $\mathrm{In}_{0.83} \mathrm{Al}_{0.17} \mathrm{~N}$ sample, as shown in Figs. 3(c) and 3(d). On further increasing the $\mathrm{Al}$ content to to AIP license or copyright; see http://apl.aip.org/apl/copyright.jsp 
$41 \%\left(\operatorname{In}_{0.59} \mathrm{Al}_{0.41} \mathrm{~N}\right)$, the bulk Fermi level and surface Fermi level are virtually coincident in energy, leading to almost no electron accumulation, as shown in Figs. 3(e) and 3(f). A reduction of the amount of electron accumulation upon increasing the $\mathrm{Al}$ content in In-rich $n$-InAlN alloys has therefore been observed, as was previously observed upon increasing the Ga content in In-rich $n$-InGaN alloys. ${ }^{8,10}$

With further increase in $\mathrm{Al}$ content, however, the samples become insulating, as revealed by single-field Hall effect measurements. This can be largely understood within the amphoteric defect model, ${ }^{25}$ whereby the favorable charge state of native defects is determined by the position of the Fermi level relative to the CNL, so that native defects act to drive the Fermi level toward the CNL. As the Al composition increases, the CNL moves closer toward the middle of the direct band gap, causing the Fermi level to become lower in the band gap, leading to insulating samples. The surface Fermi levels also tend toward the mid gap energy, suggesting approximately flatband behavior, for the Al-rich samples. Small amounts of band bending may be present at these surfaces dependent on the exact relative position of the bulk and surface Fermi levels, but this is not expected to be a large effect. This can be understood by analogy with the behavior observed at $n$ - and $p$-type InGaN surfaces, ${ }^{8,10}$ shown in the inset of Fig. 2. For $n$-type InGaN alloys, an increase in surface Fermi level to above the CNL (although below the CBM for the most Ga-rich samples) with increasing Ga content was observed, leading to electron depletion at Ga-rich surfaces. In contrast, for $p$-type $\mathrm{InGaN}$ alloys, an increase in $\mathrm{Ga}$ content was accompanied by a lowering of the surface Fermi level position further below the CNL, toward the VBM, leading to hole depletion layers. For the insulating Al-rich InAlN samples, the bulk Fermi levels will lie between that of the $n$ and $p$-type cases, leading to approximately midgap pinning of the surface Fermi level, somewhat between the two extremes observed for Ga-rich InGaN alloys, and approximately flatband conditions. However, if the Al-rich samples were doped to become $n$-type ( $p$-type), electron (hole) depletion layers would still be expected to occur at the surface, by analogy with InGaN.

In conclusion, the surface electronic properties of undoped $\operatorname{In}_{x} \mathrm{Al}_{1-x} \mathrm{~N}$ alloys have been investigated using highresolution $\mathrm{X}$-ray photoemission spectroscopy and single-field Hall effect measurements. For InN, an extreme electron accumulation was observed, consistent with previous observations. This was reduced with increasing $\mathrm{Al}$ content for the In-rich alloys, with approximately flatbands observed for $x=0.59$. Upon further increase in $\mathrm{Al}$ content, the samples became insulating, resulting in the surface Fermi level pinning close to the midgap energy with little surface spacecharge characteristics. The charge neutrality level was identified as important for determining both the bulk and surface electronic properties of the InAlN alloys across the composition range.
We are grateful to D. Law and G. Beamson of NCESS for their assistance with XPS measurements and to T. Wang and P. Parbrook of the University of Sheffield, UK, for providing the AlN sample. Also, we acknowledge the Engineering and Physical Sciences Research Council, UK, for financial support under Grant No. EP/C535553/1 and for access to the NCESS facility under Grant No. EP/E025722/1.

${ }^{1}$ H. Lu, W. J. Schaff, L. F. Eastman, and C. E. Stutz, Appl. Phys. Lett. 82, 1736 (2003).

${ }^{2}$ I. Mahboob, T. D. Veal, C. F. McConville, H. Lu, and W. J. Schaff, Phys. Rev. Lett. 92, 036804 (2004).

${ }^{3}$ D. C. Tsui, Phys. Rev. B 4, 4438 (1971).

${ }^{4}$ M. Noguchi, K. Hirakawa, and T. Ikoma, Phys. Rev. Lett. 66, 2243 (1991).

${ }^{5}$ P. D. C. King, T. D. Veal, P. H. Jefferson, S. A. Hatfield, L. F. J. Piper, C. F. McConville, F. Fuchs, J. Furthmüller, F. Bechstedt, H. Lu, and W. J. Schaff, Phys. Rev. B 77, 045316 (2008).

${ }^{6}$ C. A. Mead and W. G. Spitzer, Phys. Rev. Lett. 10, 471 (1963).

${ }^{7}$ R. E. Jones, K. M. Yu, S. X. Li, W. Walukiewicz, J. W. Ager III, E. E. Haller, H. Lu, and W. J. Schaff, Phys. Rev. Lett. 96, 125505 (2006).

${ }^{8}$ T. D. Veal, P. H. Jefferson, L. F. J. Piper, C. F. McConville, T. B. Joyce, P. R. Chalker, L. Considine, H. Lu, and W. J. Schaff, Appl. Phys. Lett. 89, 202110 (2006)

${ }^{9}$ P. D. C. King, T. D. Veal, P. H. Jefferson, C. F. McConville, H. Lu, and W. J. Schaff, Phys. Rev. B 75, 115312 (2007).

${ }^{10}$ P. D. C. King, T. D. Veal, H. Lu, P. H. Jefferson, S. A. Hatfield, W. J. Schaff, and C. F. McConville, Phys. Status Solidi B 245, 881 (2008).

${ }^{11}$ C. F. Shih, N. C. Chen, P. H. Chang, and K. S. Liu, Jpn. J. Appl. Phys., Part 1 44, 7892 (2005).

${ }^{12}$ P. D. C. King, T. D. Veal, P. H. Jefferson, C. F. McConville, T. Wang, P. J. Parbrook, H. Lu, and W. J. Schaff, Appl. Phys. Lett. 90, 132105 (2007).

${ }^{13}$ J. Wu, W. Walukiewicz, W. Shan, K. M. Yu, J. W. AgerIII, S. X. Li, E. E. Haller, H. Lu, and W. J. Schaff, J. Appl. Phys. 94, 4457 (2003).

${ }^{14}$ I. Vurgaftman and J. R. Meyer, J. Appl. Phys. 94, 3675 (2003).

${ }^{15}$ W. J. Schaff, H. Lu, L. F. Eastman, W. Walukiewicz, K. M. Yu, S. Keller, S. Kurtz, B. Keyes, and L. Gevilas, in State-of-the-Art Program on Compound Semiconductors XLI and Nitride and Wide Bandgap Semiconductors for Sensors, Photonics, and Electronics $V$, The Electrochemical Society Proceedings Series, Honolulu, Hawaii, 3-8 October 2004, edited by H. M. Ng and A. G. Baca (Electrochemical Society, Pennington, NJ, 2004), Vol. 2004-2006, pp. 358-371.

${ }^{16}$ E. Dimakis, A. Georgakilas, M. Androulidaki, K. Tsagaraki, G. Kittler, F. Kalaitzakis, D. Cengher, E. Bellet-Amalric, D. Jalabert, and N. T. Pelekanos, J. Cryst. Growth 251, 476 (2003).

${ }^{17}$ T. Wang, J. Bai, P. J. Parbrook, and A. G. Cullis, Appl. Phys. Lett. 87, 151906 (2005)

${ }^{18}$ S. A. Chambers, T. Droubay, T. C. Kaspar, and M. Gutowski, J. Vac. Sci. Technol. B 22, 2205 (2004)

${ }^{19}$ E. O. Kane, J. Phys. Chem. Solids 1, 249 (1957).

${ }^{20}$ W. Mönch, J. Appl. Phys. 80, 5076 (1996).

${ }^{21}$ R. Goldhahn, P. Schley, A. Winzer, G. Gobsch, V. Cimalla, O. Ambacher, M. Rakel, C. Cobet, N. Esser, H. Lu, and W. J. Schaff, Phys. Status Solidi A 203, 42 (2006).

${ }^{22}$ W. Mönch, Semiconductor Surfaces and Interfaces (Springer, Berlin, 2001).

${ }^{23}$ J.-P. Zöllner, H. Übensee, G. Paasch, T. Fiedler, and G. Gobsch, Phys. Status Solidi B 134, 837 (1986).

${ }^{24}$ P. D. C. King, T. D. Veal, and C. F. McConville, Phys. Rev. B 77, 125305 (2008).

${ }^{25}$ W. Walukiewicz, J. Vac. Sci. Technol. B 5, 1062 (1987). 